Cite this: Phys. Chem. Chem. Phys., 2013,

\title{
Local electronic structure of aqueous zinc acetate: oxygen K-edge X-ray absorption and emission spectroscopy on micro-jets
} 15,8046

Received 14th February 2013, Accepted 16th April 2013

DOI: $10.1039 /$ c3cp50686j

www.rsc.org/pccp

Oxygen K-edge X-ray absorption, emission, and resonant inelastic $X$-ray scattering spectra were measured to site selectively gain insights into the electronic structure of aqueous zinc acetate solution. The character of the acetate ion and the influence of zinc and water on its local electronic structure are discussed.

The acetate ion plays an important role in reactions involving a wide variety of biochemical mechanisms. ${ }^{1,2}$ In order to investigate the chemical behavior of acetate in solution, spectroscopic measurements with a high sensitivity and applicability in the aqueous phase are required to probe the local electronic structure. While X-ray absorption spectroscopy (XAS) probes the unoccupied molecular orbitals (MOs), X-ray emission spectroscopy (XES) probes the valence occupied MOs. Both methods have the capability of investigating site specific electronic states. Using XES the valence orbitals of a specified atom can be probed by tuning the excitation to a specific resonance of an atomic site under investigation. The latter method is known as resonant inelastic X-ray scattering (RIXS). ${ }^{3}$ Oxygen K-edge XAS was combined with $a b$ initio and molecular dynamics simulations revealing the strength of ion pairing with the carboxylate group. ${ }^{4}$ Furthermore, XES was used to investigate the local electronic structure of the acetate group in aqueous solution. ${ }^{5}$ In these previous studies the liquid samples were flowed behind silicon nitride membranes. Due to the recent developments in liquid micro-jets and efficient differential pumping systems combined with cryo traps, applications of soft X-ray spectroscopy techniques for studying local electronic structure and molecular bonding networks in solution have been made possible avoiding membrane

\footnotetext{
${ }^{a}$ Joint Ultrafast Dynamics Lab in Solutions and at Interfaces (JULiq),

Helmholtz-Zentrum Berlin für Materialien und Energie, Albert-Einstein-Strasse 15, 12489 Berlin, Germany

${ }^{b}$ Max-Born-Institut, Max Born Straße 2A, 12489 Berlin, Germany

${ }^{c}$ Research Center for Nanoscale Structure of Matter, Southern Federal University,

Sorge 5, Rostov-na-Donu 344090, Russia

${ }^{d}$ Freie Universität Berlin, Arnimallee 14, 14195 Berlin, Germany.

E-mail: emad.aziz@helmholtz-berlin.de; Fax: +49 308062 14757;

Tel: +4930806215003
}

effects. $^{6-15}$ Recently, we reported XA, XE and RIXS spectra measured on a micro-jet beam of pure water and wateracetonitrile mixtures at the oxygen K-edge in order to study the local electronic structure, hydrogen bond formation and cleavage dynamics. ${ }^{8,14}$ Here we further extend the use of these methods to address the local electronic structure of aqueous zinc acetate $\mathrm{Zn}\left(\mathrm{CH}_{3} \mathrm{COO}\right)_{2} \cdot\left(\mathrm{H}_{2} \mathrm{O}\right)_{n}$ solution at the oxygen K-edge. Zinc acetate has an acetate group where the oxygen K-edge lies energetically lower than the oxygen of water. ${ }^{5,16}$ Therefore, it is a good model system to study the effect of water and the $\mathrm{Zn}^{2+}$ counter cation on the carboxylate group of acetate as well as the effect of acetate on the local electronic structure of water. Detailed information about the spectrometer is presented elsewhere ${ }^{7}$ and also briefly in the Experimental section.

The oxygen K-edge XAS spectrum of $1 \mathrm{M}$ zinc acetate solution is shown in Fig. 1. In order to fingerprint the spectral features of the acetate group in aqueous solution, we included previously reported water ${ }^{7}$ and aqueous acetic acid ${ }^{5}$ XAS spectra. The zinc acetate XA spectrum consists of a peak located at $532.8 \mathrm{eV}$ followed by an edge at $535 \mathrm{eV}$ originating mainly from the oxygen absorption of water. ${ }^{17,18}$ The peak at $532.8 \mathrm{eV}$ is assigned to the oxygen $1 \mathrm{~s} \rightarrow \pi^{*}=\mathrm{O}$ absorption, ${ }^{5,19,20}$ and it is a suitable model showing the site selectivity of the method. The following peak at $535 \mathrm{eV}$ is associated with the pre-edge of water and overlaps with the acetate ion's $\mathrm{O}_{\mathrm{C}=\mathrm{O}} 1 \mathrm{~s} \rightarrow 3 \mathrm{p}$ absorption. ${ }^{16}$ The main edge of the water absorption is located at $537 \mathrm{eV}$ and the post-edge is at $542 \mathrm{eV}^{17,18}$ These features are repeated in the zinc acetate spectra; with this observation, the dominance of the waterrelated absorption starting with the pre-edge $(535 \mathrm{eV})$ and continuing for higher energies is evident.

In Fig. 1 the XA spectrum of aqueous acetic acid with a $\mathrm{pH}$ of 13.05 as reported by Horikawa et al. is included, ${ }^{5}$ and the spectrum is almost identical to that of aqueous zinc acetate. At pH 13.05, acetic acid is in its anionic form. In this deprotonated form it has a similar structure to that of the acetate group of dissociated zinc acetate as found in aqueous solution. The striking similarity between the spectra has its counterpart in the XE spectra and it will be analyzed further in the following sections. 


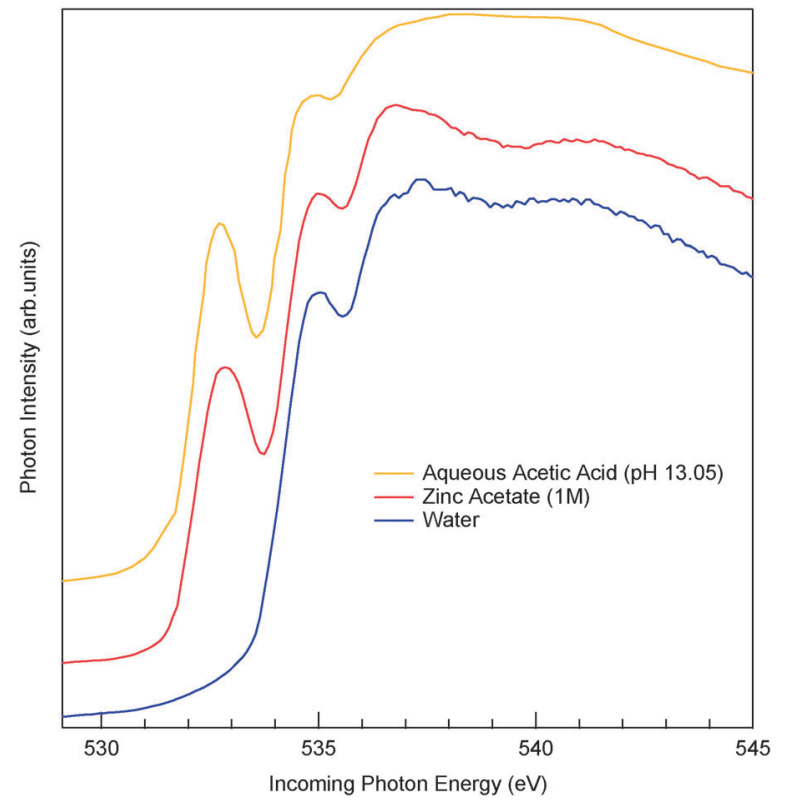

Fig. 1 K-edge XAS spectra of oxygen measured in total fluorescence yield mode. The oxygen K-edge spectrum of $1 \mathrm{M}$ aqueous zinc acetate solution is compared to those of aqueous acetic acid $^{5}$ and water. ${ }^{7}$

The series of oxygen K-edge RIXS and XE spectra of $1 \mathrm{M}$ aqueous $\mathrm{Zn}$ acetate solution are shown in Fig. 2. Upon excitation at the pre-resonances (532.8-533.9 eV), the spectra are characterized by RIXS features located at high scattering energies due to the elastic scattering. In order to find the origin of the remaining emission features located between $515 \mathrm{eV}$ and $528 \mathrm{eV}$, in Fig. 3 we compare the previously reported emission spectra of aqueous acetic acid at $\mathrm{pH}$ values of 11.44 and 5.71 (ref. 5) to those of zinc acetate aqueous solution measured at the oxygen $1 \mathrm{~s} \rightarrow \pi^{*} \mathrm{C}=\mathrm{O}$ absorption resonance of $532.8 \mathrm{eV}$. Horikawa et $a l .{ }^{5}$ performed calculations based on the density functional theory for an isolated acetate ion which we also illustrate in Fig. 3. By normalizing with respect to the highest peak, all spectra are in good agreement with each other with regard to both the higher energy region and the deeper valence orbitals and the calculation nicely presents the nature of the MOs behind each emission peak. It seems that the main spectral features in the XE spectra originate from the acetate ion itself. In a different study regarding glycine's local electronic structure, Gråsjö et al..$^{20}$ concluded that for the zwitterionic form of glycine, whose carboxylate group is deprotonated similar to the carboxylate group in our study, the main double peak structure at the energies of 525.5 and $526.6 \mathrm{eV}$ results from the emission of the out-of-plane and in-plane oxygen lone-pair orbitals, respectively. The analysis by Horikawa et al. ${ }^{5}$ increases the details of the nature of the orbitals by adding a third orbital contributing to these two features. Interestingly, a HOMO -2 (highest occupied molecular orbital) peak at $525.2 \mathrm{eV}$ and the peak related to HOMO -3 and HOMO -4 at $522.5 \mathrm{eV}$ have less intensity with respect to their counterparts in the spectrum of aqueous acetic acid when the spectra are normalized with respect to the HOMO peak as in Fig. 3. These orbitals have more

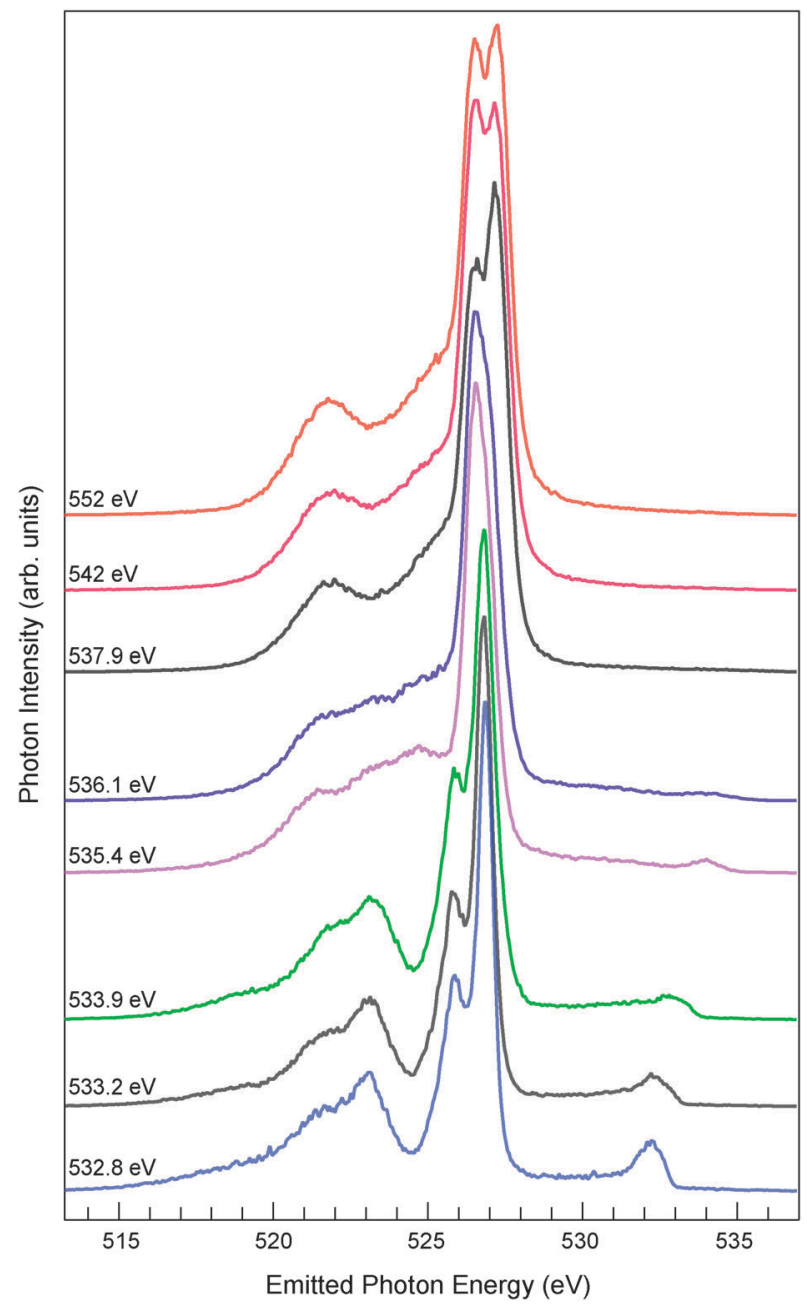

Fig. 2 Oxygen K-edge RIXS and XES spectra of $1 \mathrm{M}$ aqueous $\mathrm{Zn}$ acetate solution.

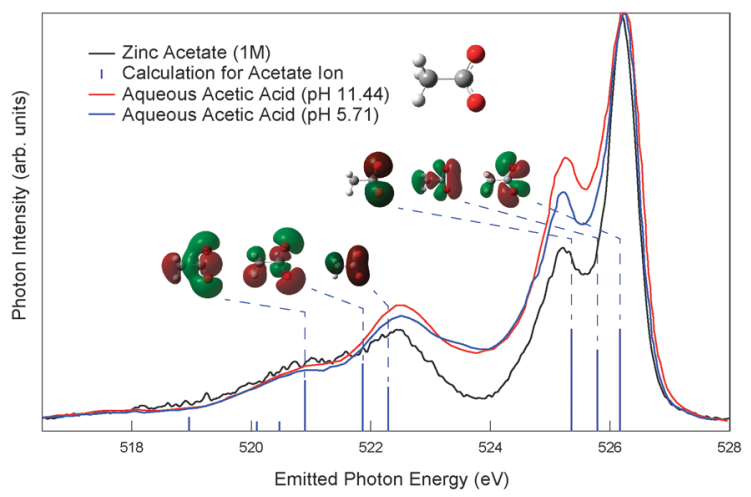

Fig. 3 Comparison of RIXS spectra of aqueous acetic acid at pH values of 11.44 and 5.71 (ref. 5) and aqueous zinc acetate solution measured at the oxygen $1 \mathrm{~s} \rightarrow \pi^{*} \mathrm{c}=\mathrm{O}$ absorption resonance at an excitation energy of $532.8 \mathrm{eV}$.

sigma character with regard to the $\mathrm{C}-\mathrm{O}$ bonds than the remaining MOs. This is the point where we have to consider the effect of the extra $\mathrm{Zn}^{2+}$ ion and water on the local electronic structure of the acetate ion. In the preparation of the aqueous acetic acid sample by Horikawa et al., ${ }^{5} \mathrm{NaOH}$ was used for the $\mathrm{pH}$ adjustment. The exact 
nature of the interaction between $\mathrm{Na}^{+}$and acetate ions is not known, nevertheless, we have clearly shown before that $\mathrm{Na}^{+}$has a strong interaction with the carboxylate group. ${ }^{4}$ Accordingly, we can propose that, the differences in the RIXS spectra shown in Fig. 3 are due to the replacement of $\mathrm{Na}^{+}$by $\mathrm{Zn}^{2+}$. Before concluding this part, the effect of possible protonation of the acetate ion in aqueous $\mathrm{Zn}$ acetate must be considered. The $\mathrm{pH}$ of $1 \mathrm{M}$ solution was measured to be 5.7 , therefore we also included the spectra of aqueous acetic acid with a similar $\mathrm{pH}$ value for comparison. In this region, $12 \%$ of the acetic acid molecules is in neutral form causing the aforementioned peak structures to lose intensity. ${ }^{5}$ This effect cannot be ruled out in the quenching of the peaks in aqueous $\mathrm{Zn}$ acetate together with the native oxide emission due to the membrane mentioned in a recent study by the same group. ${ }^{21}$ To draw a clearer conclusion with a quantitative analysis, a further study is planned to investigate the effects of different counter cations on the carboxylate group.

In Fig. 4, we present the mutual effect of water and the carboxylate group on the local electronic structure of each other. The figure consists of a comparison of the evolution of the emission spectra of zinc acetate and water with increasing excitation energies. The series of spectra starts with an excitation energy of $533.9 \mathrm{eV}$ which sits on the higher energy flank of the $\mathrm{O}_{\mathrm{C}=\mathrm{O}}$ resonance of acetate, and ends with $542 \mathrm{eV}$ which is post-resonance for both of the species. The series starts with a spectrum substantially characterized by the acetate ion, which evolves towards a water emission spectrum and becomes almost identical to it at the post-resonance. This observation is in line with Gråsjö et al.'s conclusion regarding the aqueous glycine study. ${ }^{20}$ Among these five pairs of spectra, the fourth pair at an excitation energy of $537.9 \mathrm{eV}$ requires a special emphasis because at this excitation energy an interesting phenomenon emerges. The peak splitting in water XE spectra at the main peak at around $526.5 \mathrm{eV}$, which is associated with the water's oxygen lone-pair character orbital $1 b_{1},{ }^{2-24}$ is commonly attributed to the sensitivity towards hydrogen bonding. ${ }^{8,14,22-25}$ Based on our previous studies, the left feature is referred to as the $d_{2}$ peak, the right one as $d_{1}$, and the relative intensity of these peaks with respect to each other is considered as a fingerprint for the strength of the hydrogen bonding. As the relative intensity of the $d_{2}$ peak decreases, it is inferred that the hydrogen bonding network is disrupted and the number of HB's decreases. We observe this signature and peak quenching at an excitation energy of $537.9 \mathrm{eV}$; the relative intensity of the $\mathrm{d}_{2}$ peak decreases with respect to the $d_{1}$ peak. This is in line with the expectation that, due to the introduction of $\mathrm{Zn}^{2+}$ and acetate ions into the water network, the number of HB's will decrease. Thus, this is clear evidence for the interaction between solute and solvent and hence their electronic configurations.

Interestingly, at higher excitation energy, namely $542 \mathrm{eV}$, the $\mathrm{XE}$ spectra of pure water and aqueous zinc acetate become almost identical. The post-edge of water's XA spectra is sensitive to the hydrogen bonding features, which is reflected at an excitation energy of $537.9 \mathrm{eV} .^{26}$ However, it is observed that at an excitation energy of $542 \mathrm{eV}$, the sensitivity of the XE spectra with regard to the hydrogen bonding strength decreases.

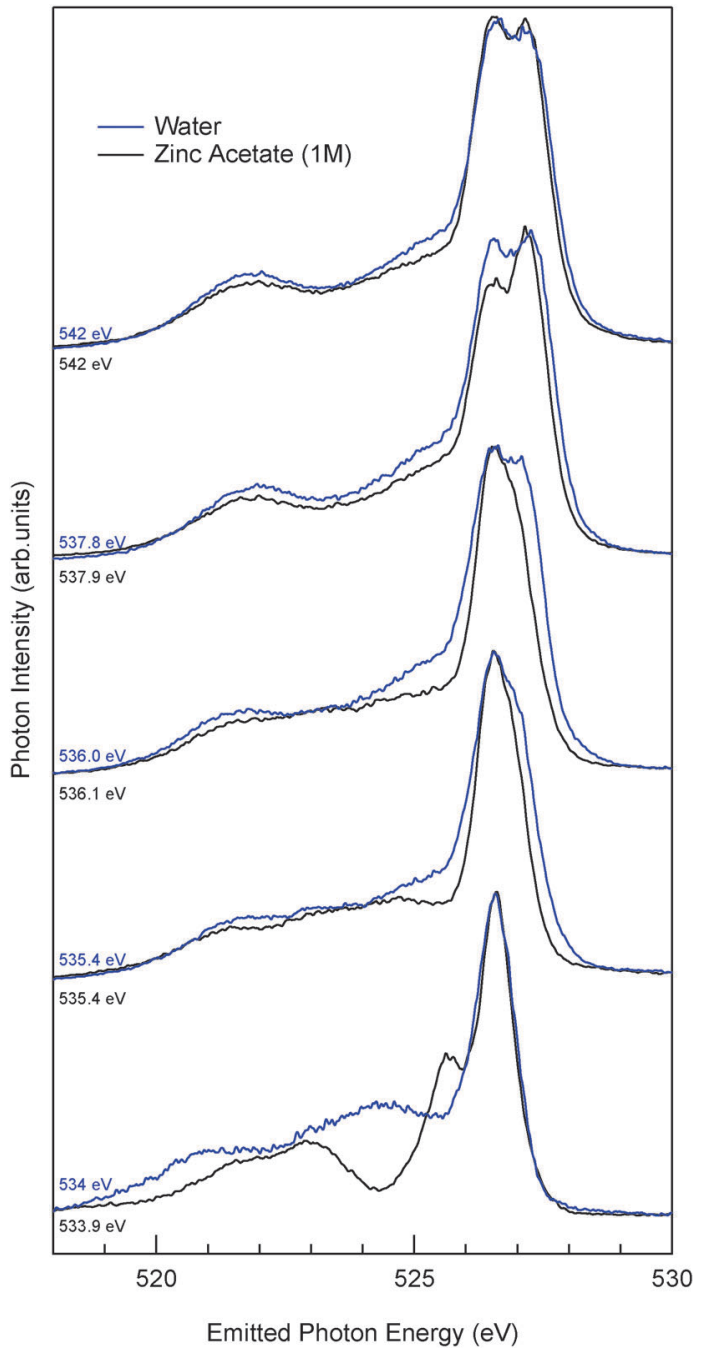

Fig. 4 Comparison of the RIXS spectra of $1 \mathrm{M}$ aqueous zinc acetate solution and pure water at similar excitation energies.

\section{Conclusion}

Oxygen K-edge absorption and emission spectra of $1 \mathrm{M}$ aqueous zinc acetate solution were measured using our recently developed $\mathrm{X}$-ray emission spectrometer on micro-jets. We compared the $\mathrm{Zn}^{2+}$ counter cation to the previously studied $\mathrm{Na}^{+}$counter cation ${ }^{5}$ and fingerprinted the countercation effect on the HOMOs of the carboxylate group. The effect was rather pronounced on HOMO -2 , HOMO -3 and HOMO -4 which have more sigma character with regard to the $\mathrm{C}-\mathrm{O}$ bonds than the remaining MOs. On the other hand, the effect of the carboxylate group on the local electronic structure of water is observed in the emission spectrum at the excitation energy of the post-edge absorption of water which is referred to for its sensitivity to HB coordination.

\section{Experimental}

Detailed information about the LixEdrom setup is presented elsewhere. ${ }^{7}$ Briefly, the experimental station is equipped with a liquid micro-jet using a capillary with a $20 \mu \mathrm{m}$ nozzle that 
produces $\sim 10 \mathrm{~mm}$ laminar flow of liquid beams in vacuum. The high vacuum conditions required for soft X-ray experiments are preserved by differential pumping sections and liquid nitrogen cooling traps that reduce the effective pressure by several orders of magnitude. The pressure in the experimental chamber is generally around $10^{-5} \mathrm{mbar}$ and $<10^{-8} \mathrm{mbar}$ at the connection to the beamline. The XA spectra were recorded in total fluorescence yield (TFY) mode with a GaAsP diode. For the XE and RIXS measurements the spectrometer was used, which is currently equipped with four high energy resolution spherical gratings, together covering the entire soft X-ray energy range of 30-1200 eV, and the detector consisting of a MCP-stack and a CCD camera is operated at a base pressure of $c a \cdot 10^{-8} \mathrm{mbar}$.

The XES spectrometer was operated in normal scattering geometry and the synchrotron beam was linearly polarized. The small focus of the synchrotron beam at the U41 beamline is very well-suited for experiments with the liquid micro-jet; the sizes of the jet and X-ray beam nicely match.

Zinc acetate with $99.5 \%$ purity was obtained from Merck and used without further purification. The $\mathrm{pH}$ value for the $1 \mathrm{M}$ solution was measured to be 5.7 .

\section{Acknowledgements}

This work was supported by the Helmholtz-Gemeinschaft via the young investigator fund VH-NG-635. Part of this work was financially supported by the European Research Council grant no. 279344. K.A. would like to acknowledge the financial support from the Einstein Foundation Berlin for the postdoctoral scholarship awarded to the Aziz team.

\section{Notes and References}

1 R. Hovik, B. Brodal, K. Bartlett and H. Osmundsen, J. Lipid Res., 1991, 32, 993-999.

2 W. C. Hallows, S. Lee and J. M. Denu, Proc. Natl. Acad. Sci. U. S. A., 2006, 103, 10230-10235.

3 F. Gel'mukhanov and H. Agren, Phys. Rep., 1999, 312, 87-330.

4 E. F. Aziz, N. Ottosson, S. Eisebitt, W. Eberhardt, B. JagodaCwiklik, R. Vacha, P. Jungwirth and B. Winter, J. Phys. Chem. B, 2008, 112, 12567-12570.

5 Y. Horikawa, T. Tokushima, Y. Harada, O. Takahashi, A. Chainani, Y. Senba, H. Ohashi, A. Hiraya and S. Shin, Phys. Chem. Chem. Phys., 2009, 11, 8676-8679.

6 R. Seidel, S. Ghadimi, K. M. Lange, S. Bonhommeau, M. A. Soldatov, R. Golnak, A. Kothe, R. Könnecke, A. Soldatov, S. Thürmer, B. Winter and E. F. Aziz, J. Am. Chem. Soc., 2012, 134, 1600-1605.

7 K. M. Lange, R. Könnecke, S. Ghadimi, R. Golnak, M. A. Soldatov, K. F. Hodeck, A. Soldatov and E. F. Aziz, Chem. Phys., 2010, 377, 1-5.
8 K. M. Lange, R. Könnecke, M. Soldatov, R. Golnak, J. E. Rubensson, A. Soldatov and E. F. Aziz, Angew. Chem., Int. Ed., 2011, 50, 10621-10625.

9 K. M. Lange, A. Kothe and E. F. Aziz, Phys. Chem. Chem. Phys., 2012, 14, 5331-5338.

10 B. Winter, Nucl. Instrum. Methods Phys. Res., Sect. A, 2009, 601, 139-150.

11 B. Winter, E. F. Aziz, U. Hergenhahn, M. Faubel and I. V. Hertel, J. Chem. Phys., 2007, 126, 124504.

12 B. Winter, E. F. Aziz, N. Ottosson, M. Faubel, N. Kosugi and I. V. Hertel, J. Am. Chem. Soc., 2008, 130, 7130-7138.

13 E. F. Aziz, N. Ottosson, M. Faubel, I. V. Hertel and B. Winter, Nature, 2008, 455, 89-91.

14 K. M. Lange, M. Soldatov, R. Golnak, M. Gotz, N. Engel, R. Könnecke, J. E. Rubensson and E. F. Aziz, Phys. Rev. B: Condens. Matter Mater. Phys., 2012, 85, 155104.

15 E. F. Aziz, J. Phys. Chem. Lett., 2011, 2, 320-326.

16 T. Tokushima, Y. Horikawa, Y. Harada, O. Takahashi, A. Hiraya and S. Shin, Phys. Chem. Chem. Phys., 2009, 11, 1679-1682.

17 P. Wernet, D. Nordlund, U. Bergmann, M. Cavalleri, M. Odelius, H. Ogasawara, L. A. Näslund, T. K. Hirsch, L. Ojamae, P. Glatzel, L. G. M. Pettersson and A. Nilsson, Science, 2004, 304, 995-999.

18 D. E. Smith, C. D. Cappa, B. M. Messer, W. S. Drisdell, R. C. Cohen and R. J. Saykally, J. Phys. Chem. B, 2006, 110, 20038-20045.

19 B. M. Messer, C. D. Cappa, J. D. Smith, K. R. Wilson, M. K. Gilles, R. C. Cohen and R. J. Saykally, J. Phys. Chem. $B, 2005,109,5375-5382$.

20 J. Gråsjö, E. Andersson, J. Forsberg, L. Duda, E. Henke, W. Pokapanich, O. Bjorneholm, J. Andersson, A. Pietzsch, F. Hennies and J. E. Rubensson, J. Phys. Chem. B, 2009, 113, 16002-16006.

21 T. Tokushima, Y. Horikawa, H. Arai, Y. Harada, O. Takahashi, L. G. M. Pettersson, A. Nilsson and S. Shin, J. Chem. Phys., 2012, 136, 044517.

22 O. Fuchs, M. Zharnikov, L. Weinhardt, M. Blum, M. Weigand, Y. Zubavichus, M. Bar, F. Maier, J. D. Denlinger, C. Heske, M. Grunze and E. Umbach, Phys. Rev. Lett., 2008, 100, 027801.

23 T. Tokushima, Y. Harada, Y. Horikawa, O. Takahashi, Y. Senba, H. Ohashi, L. G. M. Pettersson, A. Nilsson and S. Shin, J. Electron Spectrosc. Relat. Phenom., 2010, 177, 192-205.

24 T. Tokushima, Y. Harada, O. Takahashi, Y. Senba, H. Ohashi, L. G. M. Pettersson, A. Nilsson and S. Shin, Chem. Phys. Lett., 2008, 460, 387-400.

25 N. Engel, K. Atak, K. M. Lange, M. Gotz, M. Soldatov, R. Golnak, E. Suljoti, J.-E. Rubensson and E. F. Aziz, J. Phys. Chem. Lett., 2012, 3697-3701.

26 A. Nilsson and L. G. M. Pettersson, Chem. Phys., 2011, 389, $1-34$. 